\title{
Requisitos Básicos para la Implementación de un servicio Farmacéutico de Preparaciones Magistrales
}

Basic requirements for the implementation of a pharmaceutical compounding service.

Dr. Luis Carlos Monge Bogantes ${ }^{1}$ MSc

Recibido: 15-05-19

Mauricio Abel Chavarria²

Aceptado: 10-06-19

1- Farmacéutico, docente universitario en UCIMED.

2- Estudiante de farmacia,UCIMED.

\section{Resumen:}

Las preparaciones magistrales de formulaciones no estériles son de las más comunes que se conocen y establecidas por muchos regímenes y órganos del ámbito farmacéutico a nivel global. En este artículo se guiará a los lectores por los principios fundamentales de las preparaciones magistrales de fórmulas no estériles, lo cual es de suma importancia para iniciar con conocimientos en el entorno de las ciencias farmacéuticas que conlleva muchas responsabilidades con el fin del bienestar de las y los usuarios de los medicamentos magistrales que necesitan de la ayuda de las y los farmacéuticos.

\section{Palabras claves:}

Farmacia, Fórmulas no estériles, Preparaciones magistrales, medicamentos no estériles.

\section{Abstract:}

Compounding of non-sterile formulations are among the most common that are known and established by many regimens and organs of the pharmaceutical field at a global level. In this article the readers will be guided by the fundamental principles of the masterful preparations of non-sterile formulas, which is of the utmost importance to start with knowledge in the pharmaceutical sciences environment that entails many responsibilities for the welfare of the users of the master drugs that need the help of the pharmacists.

Keywords: Pharmacy, compounding, non-sterile formula, non-sterile medication.

El presente artículo, describe la información sobre la implementación de Preparaciones Magistrales en los términos de No estériles y, con el fin de obtener una síntesis de la mejor información, métodos, prácticas y ensayos sobre lo más requerido para estudiantes y profesionales en farmacia, docentes o personas interesadas en las ciencias farmacéuticas para que obtengan un amplio conocimiento sobre dichas formulaciones. Cabe destacar que la información obtenida de referencia ha sido indagada profundamente por artículos y documentos de gran calidad, que contiene bastante información valida sobre las preparaciones magistrales de formulación no estériles.

Conceptos de formulaciones no estériles: es una preparación farmacéutica que se puede formular y elaborar en condiciones ambientales controladas, pero no estériles.

Descripción de categorías de preparaciones magistrales No estériles: 
- Simple: Categoría que contiene descripciones de las preparaciones magistrales en una Farmacopea, o que contenga información de un artículo de una publicación de referencia que abarca equipo preparación, procedimiento, FLU (Fecha de Límite de Uso), datos de estabilidad de cada formulación, manejo de productos comerciales farmacéuticos y otros ámbitos.

- Moderada: Fabricación de una preparación farmacéutica que necesite de cálculos o métodos especiales para evaluar las cantidades de componentes de cada preparación y cada unidad de dosificación, también se puede calcular para preparaciones que no tengan datos de estabilidad para su formulación específica respectiva.

- Compleja: Es la preparación que se elabora con requerimientos de capacitación, instalaciones, equipo, procedimientos especiales y las áreas de trabajo en que se elaboran las preparaciones, todo con el fin de asegurar los resultados terapéuticos adecuados. (The United States Pharmacopeial Convention, 2017).

- Responsabilidades del Preparador: EI profesional farmacéutico debidamente certificado que elabora las preparaciones tiene una gran responsabilidad en tener sus preparaciones magistrales con calidad, seguro y eficacia. Tiene otras responsabilidades que son dispensar la preparación finalizada, con su respectivo etiquetado, envasado, conforme a los requisitos escritos por agencias estatales, legislaciones nacionales e internacionales.

Los profesionales en farmacia que elaboren las preparaciones magistrales, de medicamentos, deben tener una competencia en sus trabajos, además de desarrollar más sus conocimientos sobre sus ámbitos de ejercicio laboral. (The United States Pharmacopeial Convention, 2017).

Principios Generales de Preparación Magistral de formulación No estéril:

- El personal debe estar obligatoriamente capacitado, la capacitación debe estar documentada.

- Los elementos de las sustancias o ingredientes de la preparación magistral deben tener su identidad del producto, calidad asegurada, pureza, almacenamiento seguro y que todos los productos y la información se obtenga de compañías confiables.

- Examinación de los apartados de la prescripción médica, su información debe estar completa y ser legible.

- Todos los instrumentos deben estar sanitizados, limpios, y que requieran de un buen mantenimiento adecuado.

- En entorno donde se realiza la preparación magistral debe ser seguro y limpio, especialmente cuando se elabora preparaciones de fármacos.

- Solo puede elaborar el personal autorizado en las operaciones de preparación.

- Se debe examinar todos los datos de identificación de la preparación magistral, datos del paciente de manera legible en la etiqueta que trae la preparación. (Solís, 2018).

Formas farmacéuticas No estériles:

Son preparaciones que permiten la mínima presencia de microrganismos como bacterias, conforme señale las restricciones y limitaciones de la farmacopea que se diseñan, formulan y producen para uso de vía oral u tópica. A continuación, se obtiene un cuadro de las formas farmacéuticas y sus tipos:
Forma Farmacéutica:

Sólido orales

Semisólidos
Tipo de forma farmacéutica:

Grageas, Comprimidos y cápsulas

Cremas, ungüentos, supositorios y óvulos

Jarabes, suspensiones y gotas.

Tabla 1. Formas farmacéuticas y su tipo, (Solís, 2018). 
1. Criterios para Elaboración de una Preparación Magistral para cada Fármaco:

- $\quad$ Revisión de las dosis, la seguridad y el uso previsto de la preparación en términos:

o Propiedades químicas y físicas de los componentes.

o Forma farmacéutica.

- Uso terapéutica y vía de administración (incluyendo disposición biológica local y sistémica). o Limitaciones legales, en el caso de hallarse.

- $\quad$ Crear Registro Maestro de formulación.

- $\quad$ Ingredientes deben tener identidad, calidad y pureza esperadas.

- $\quad$ Preparaciones magistrales se elabora en área limpia y sanitizada.

- Seleccionar el equipo de preparación magistral adecuado para cada preparación.

- $\quad$ Establece FLU (Fecha de Límite de Uso) indicada en el etiquetado.

- $\quad$ Personal de trabajo con higiene y vestimenta adecuada para preparaciones magistrales.

- La preparación se elabora con información y datos científicos.

- $\quad$ El farmacéutico formulador verifica y pone atención a procesos críticos.

- La preparación final se evalúa con factores del peso, potencia, mezclado, olor, color, claridad, consistencia, $\mathrm{pH}$ y análisis analíticos. La información se coloca en el Registro de Preparación Magistral.

- La preparación se envasa según la sección de Envases de Preparaciones Magistrales.

- El envase de preparación se etiqueta conforme a las legislaciones estatales y federales aplicables. El etiquetado debe decir "Ésta es una preparación magistral."

- $\quad$ El preparador debe revisar el RMF (Registro Maestro de Formulación) y RPM (Registro de Preparación Magistral) para asegurar que no exista algún error.

- La preparación se entrega al paciente o proveedor de cuidados y precauciones con la consulta farmacéutica adecuada. (The United States Pharmacopeial Convention, 2017).

2. Instalaciones de Preparación Magistral:

Las instalaciones de preparación magistral tienen que contener un área apropiada, determinando el diseño para preparar cada prescripción y forma farmacéutica no estéril específica. Todos los artefactos e instrumentos deben estar colocados en orden para prevenir confusiones (envases, ingredientes, etiquetas, materiales en proceso y producto terminado). Las áreas de preparaciones estériles deben estar separadas y ser diferentes a las preparaciones de no estériles. Otros requisitos importantes son el proveer agua potable calidad USP en el lavado de mano y equipo, un sistema de cañería que debe estar libre de alguna irregularidad que pueda provocar la contaminación en el área de preparaciones magistrales, se debe tener una excelente iluminación, también se debe tener un sistema de calefacción y ventilación aislados del resto del área para evitar la contaminación y descomposición de los productos, se debe monitorear la humedad, presión y temperatura según requiera cada producto (no mayor a 25 grados centígrados en nuestro país).

Los fármacos peligrosos deben almacenarse, distribuirse y prepararse por profesionales farmacéuticos debidamente capacitados para estos casos de condiciones de trabajo de alto riesgo, todo con el fin de manipular correctamente los fármacos peligrosos y proteger la seguridad de los trabajadores y los pacientes. (The United States Pharmacopeial Convention, 2017).

\section{Equipo de Elaboración de las preparaciones magistrales:}

Los artefactos e instrumentos usados deben tener un tipo de diseño y uso adecuado, para no afectar a la pureza de los ingredientes y productos de modo que el equipo y los ingredientes no reaccionen, no adicionen ni absorban a dichos productos.

También el equipo debe estar almacenado, y mantenerse limpio por un personal de limpieza debidamente entrenado. Los equipos automatizados ya sean mecánicos, electrónicos $u$ otros tipos deben ser inspeccionados constantemente para que no existan fallas en sus funciones. 
llustración 1. Equipo y artefactos del laboratorio en fórmulas no estériles. (PCE- Ibéricas, s.f.)

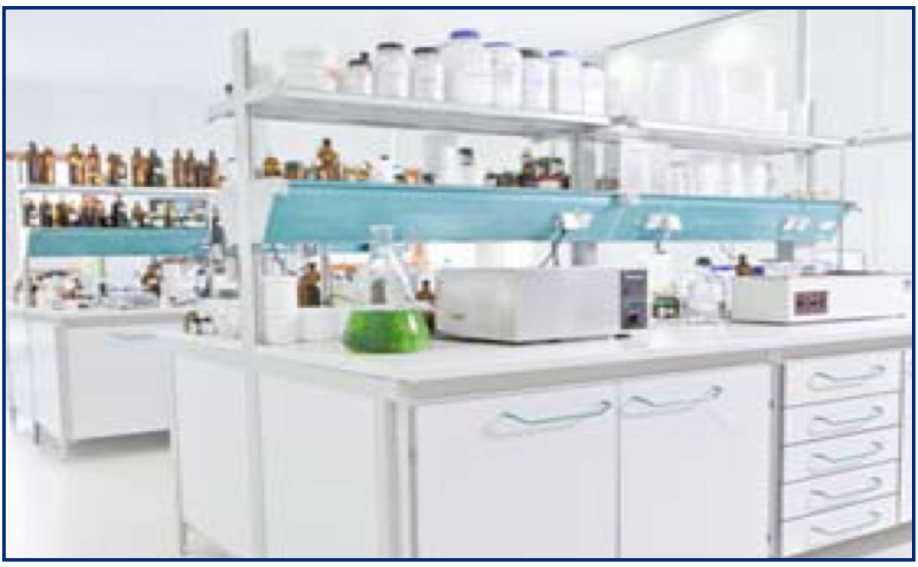

4. Manejo y distribución de los componentes:

- Se aconseja verificar la calidad de una sustancia en una Farmacopea de su respectiva región.

- Los elaboradores de componentes, deben usar sustancia fabricadas en una institución registrada de administración y verificación de medicamentos como es la FDA (Food and Drug Administration).

- $\quad$ Las preparaciones magistrales oficiales se preparan de ingredientes individuales de calidad y que cumplan los requisitos de la monografía oficial, se pueden etiquetar con siglas USP o NF según corresponda.

- $\quad$ Cuando la preparación tiene fines de uso nutricional o dietéticos, el preparador debe cumplir con los requisitos legales.

- Las elaboraciones de preparaciones magistrales en humanos, el preparador debe consultar la lista de componentes extraído del mercadodeunainstitución seguradeadministración y elaboración de medicamentos como el caso de la FDA (Food and Drug Administration) por razones de seguridad de los productos, en caso de elaboración de preparaciones magistrales en Animales con lo que se producen alimentos, el preparador debe consultar la lista de restricciones de componentes para su uso animal, con lo que producen los alimentos.

- Todas las sustancias, fármacos y medicamentos deben almacenarse y distribuirse según diga las instrucciones de los preparadores de acuerdo a los requerimientos de la monografía USP, NF o FCC.

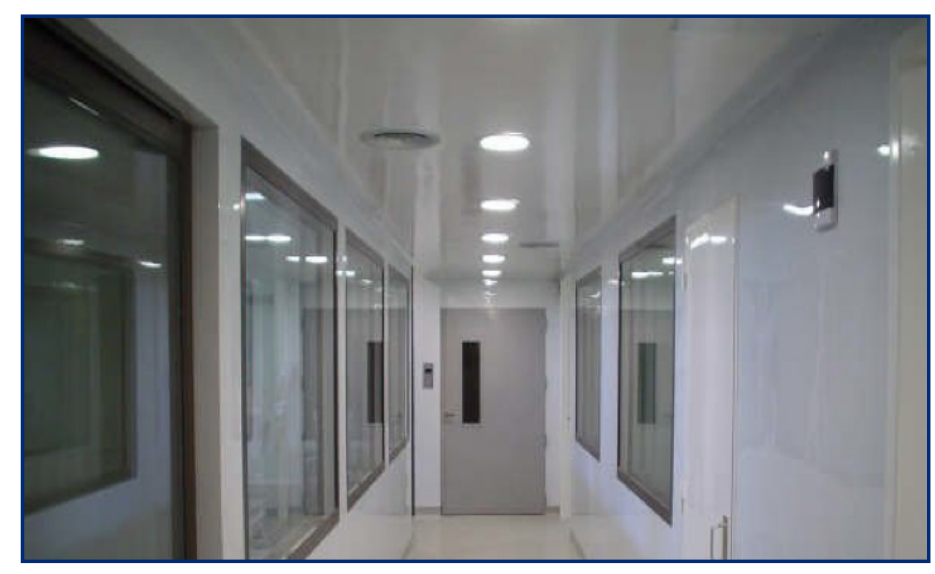

Ilustración 2. Pasillo de laboratorio de formulaciones no estériles. (Cuben, s.f.)

5. Criterios de Estabilidad y designación de Fecha Límite de Uso

La FLU es la fecha en la cual la preparación no tiene que usarse más y se calcula por la elaboración del profesional farmacéutico que lo preparó. La FLU se determina según las consultas e información disponibles en documentos sobre su consistencia general y la parte de su fármaco específico, se debe considerar los siguientes factores:

- Naturaleza fármaco.

o Forma farmacéutica.

o Potencial de proliferación microbiana en su elaboración.

o El contenido de su envase.

- Condiciones y factores de almacenamiento.

o El tiempo pronosticado sobre el tratamiento.

Cuando se usan ingredientes de producto activo farmacéutico para una preparación no estéril, la fecha de expiración no se puede usar como el único factor para determinar una FLU, sino también el preparador debe referir información de estabilidad prescrita por el fabricante, para obtener información de su compatibilidad, estabilidad y degradación del componente, cabe destacar que toda la información debe estar interpretado en función con la formulación real. Siempre el farmacéutico debe visualizar si el medicamento elaborado tenga algún rasgo de inestabilidad durante su proceso de elaboración, almacenamiento, distribución, despacho y dispensación. (The United States Pharmacopeial Convention, 2017). 


\section{Envasado:}

El profesional farmacéutico que elabora la preparación magistral, debe asegurar el cierre correcto de los envases usados de dicha preparación y de que estos cumplen los requisitos farmacopeicos USP. El proveedor de envases debe proporcionar la información del cumplimiento del envase con respecto a los criterios de calidad farmacopeicos USP. Siempre los envases deben permanecer limpios, alejados del piso o áreas de residuos y almacenarse en áreas limpias.

Los envases deben ser elaborados con material limpio con el objetivo de no alterar el contenido del material con su pureza, También se considera la relación envase-fármaco para sustancia con propiedades adsortivas. (The United States Pharmacopeial Convention, 2017).

\section{Documentación sobre la preparación magistral:}

La documentación otorga al farmacéutico la oportunidad de evaluar, indagar y repetir los pasos durante el proceso de elaboración magistral, siempre cuando se requiera, y manterner una adecuada trazabilidad en todo momento. Todos los profesionales en farmacia que dispensen una receta, deben cumplir con los reglamentos de manutención de registros de sus intervenciones y de las formulaciones magistrales que dispensen.

Es obligatorio que todos los documentos posean su definición, conceder la fecha actualizada de cada uno, tener un título de portada, la escritura debe ser legible, clara y objetiva, Es importante determinar los procedimientos operativos establecidos contenga la utilización, de mantenimiento y calificación de cada uno de los artefactos e instrumentos, la gestación y control de calidad de las preparaciones magistrales. Por último, la documentación debe ser certificada y analizada por el farmacéutico que esté a cargo. (Solís, 2018).

8. Comparación de Registro Maestro de Formulación y Registro de Preparación Magistral.

Los registros maestros y registros preparación magistral deben contener:

\section{Registro Maestro de Formulación}

- Nombre contenido y forma farmacéutica de la preparación.

- Cálculos para determinar la cantidad componentes y sus dosis de ingredientes farmacéuticos activos, también describir los ingredientes y sus cantidades respectivas

- Instrucciones de mezclado que debe incluir la temperatura de mezclado y orden de mezclado.

- Información del etiquetado de la muestra que incluya: nombre genérico y cantidad concentración de cada ingrediente activo, FLU descrita, estándares de almacenamiento y con su numeración de prescripción.

- Descripción de la preparación final

- Procedimientos de control calidad con sus resultados.

\section{Registro de Preparación Magistral}

- Nombre oficial, contenido y dosis de la preparación.

- Nombre y cantidades de los componentes, también se debe incluir las fuentes, número lotes y fecha de caducidad.

- Nombre de todos los profesionales que elaboraron todo el proceso de preparación magistral.

- Documentación de cualquier incidente del control de calidad en la preparación magistral e incidentes del proveedor de información de cuidados del paciente.
- Fecha y FLU indicada.

- Descripción de la preparación magistral

- Resultados del control de calidad.

Tabla 2. Comparación de Registros Maestro Formulación y Registro de Preparación Magistral. (The United States Pharmacopeial Convention, 2017). 
9. Control de Calidad:

El control de Calidad debe contener dentro de sus estándares la seguridad, calidad y desempeño de las preparaciones magistrales de forma correcta y eficaz sobre sus procedimientos utilizados en la preparación magistral. El profesional en farmacia debe verificar cada procedimiento que se realiza durante todo el transcurso del trabajo. Al final, el preparador debe observar y analizar la preparación terminada, también debe investigar cualquier discrepancia o error para poder corregir el fallo. (The United States Pharmacopeial Convention, 2017).

\section{Consejos al paciente:}

Se debe instruir al paciente o su representante para informarles sobre el uso correcto del fármaco, también debe explicar sobre sus eventos adversos y cualquier caso si existe algún cambio físico que se pueda observar de la preparación magistral dispensada. El farmacéutico debe investigar y documentar cualquier incidente de la información que se relaciona con la preparación magistral y deberá resolverlo con estándares correctivos siguiendo los criterios de farmacovigilancia. (The United States Pharmacopeial Convention, 2017).

\section{Capacitación al profesional:}

TTodos los profesionales en farmacia deben estar instruidos con una implementación de un programa de capacitación y que se aplica constantemente. Los preparados deben ser evaluados al menos una vez en doce meses. Las capacitaciones contienen ciertas etapas que deben de realzar:

- Todos los trabajadores implicados en la preparación de fármacos deben leer e instruirse de los procedimientos de las preparaciones magistrales, que deban incluir la información de instalaciones, personal, informes de seguridad, equipo, información sobre las sustancias, envasado, almacenamiento y distribución.

- Todos los profesionales en farmacia que manipulen fármacos peligrosos, deben estar previamente capacitados en el mantenimiento, manipulación y desechos de dichos fármacos.

- Todos los procedimientos, datos de la preparación magistral y cálculos deben ser documentados.

- El farmacéutico debe realizar una demostración de los procedimientos para los asistentes o técnicos para guiarlo e instruirlo durante el proceso de capacitación que siempre deberán estar bajo su supervisión directa. Luego el asistente o técnico deberá repetir los procedimientos sin ninguna ayuda del instructor. Cuando el regente farmacéutico esté satisfecho sobre los conocimientos del asistente o técnico, él o ella deberá firmar los registros de documentación que demuestran que el empleado recibió la capacitación.

- El farmacéutico debe monitorear constantemente al empleado durante el proceso de la preparación, asegurar que los cálculos estén correctos y que los procedimientos del empleado sean correctos, el preparador es el único responsable de la finalización de la preparación elaborada. (The United States Pharmacopeial Convention, 2017).

12. Preparaciones magistrales para pacientes animales:

El farmacéutico debe diferenciar la preparación magistral para animales y humanos, por ende, todos los profesionales en farmacia que preparen fármacos o alimentos para animales, deben obtener previos conocimientos sobre la regulación y aplicación de dichos fármacos en pacientes animales. Por la ley, los veterinarios tienen la obligación de proporcionar los cuidados a los animales con lo que se producen alimentos de retención de tejidos de animales tarados como la carne, leche y huevo, antes de ingresar en el suministro alimenticio humano. El uso de fármacos en animales está regulado por los gobiernos. El farmacéutico debe ser informado de las especies específicas en su capacidad fisiológica y metabólicas que pueden ser tóxicos cuando se utiliza algunos fármacos o excipientes en preparaciones magistrales. También otro reglamento describe que los veterinarios y farmacéuticos que elaboran preparaciones, deben estar informados sobre las legislaciones estatales y federales relacionado al uso de fármacos en 